\title{
The mechanical design of the BARREL section of the detector CALIFA for $\mathbf{R}^{3}$ B-FAIR
}

\author{
E. Casarejos ${ }^{1, a}$, H. Alvarez-Pol' ${ }^{2}$, D. Cortina-Gil², I. Durán², P. Izquierdo ${ }^{1}$, P. Yañez ${ }^{1}$, and J.A. \\ Vilán ${ }^{1}$ \\ ${ }^{1}$ Dpt. of Mechanical Engineering, University of Vigo, E-36310 Vigo, Spain \\ ${ }^{2}$ Dtp. of Particle Physics, University of Santiago de Compostela, E-15782, Santiago de Compostela, Spain
}

\begin{abstract}
In this work we present the mechanical concept proposed for one of the sections of the detector CALIFA of the $\mathrm{R}^{3} \mathrm{~B}$ experiment for FAIR. The use of an alveolar structure made of carbon-fiber composites allows for a light and robust solution to hold the active elements with an extreme mass ratio below $0.7 \%$. The active core is supported by structural elements designed to make a fully operational assembly, taking care of different configurations and functionality. All the design has been developed using intensive calculation based in finite elements models and physical simulations.
\end{abstract}

\section{Introduction}

The detector CALIFA (CALorimeter for the In Flight detection of gamma-rays and light charged pArticles) is one of the key detectors of the $\mathrm{R}^{3} \mathrm{~B}$ experiment [1] at the facility FAIR [2]. FAIR (Facility for Anti-proton and Ion Research) is the next step beyond the GSI [3], developing physics programs in four main fields, one of those being Nuclear Structure, Astrophysics and Reactions. This program is driven by the so called NUSTAR collaboration, joining the efforts of some seven hundreds researchers of more than 150 institutes from all over the world.

The next generation experimental setup for studies of Reactions with Relativistic Radioactive Beams $\left(\mathrm{R}^{3} \mathrm{~B}\right)$ is one of the eight experiments of the NUSTAR collaboration, based in the use of the intense secondary nuclear beams provided by FAIR. The $\mathrm{R}^{3} \mathrm{~B}$ setup will cover experimental reaction studies with exotic nuclei far off stability with emphasis on nuclear structure and dynamics, as well as Astrophysical aspects and technical applications. The $\mathrm{R}^{3} \mathrm{~B}$ collaboration includes more than 50 different institutes, and several hundreds of collaborators.

The detector CALIFA (CALorimeter for the In Flight detection of gamma-rays and light charged pArticles) surrounds the $\mathrm{R}^{3} \mathrm{~B}$ reaction target and will be used in a wide spectrum of experiments. It will feature a high photon detection efficiency and good energy resolution even for beam energies approaching $1 \mathrm{AGeV}$, as well as the required calorimetric properties for detection of multiple gammaray cascades, and the detection of protons with good energy resolution up to several hundreds of MeVs. CALIFA consists of two sections, a cylindrical Barrel and a Forward EndCap. To minimize the effect of the Doppler broadening on the energy resolution, the segmentation must be large enough. Therefore the polar and azimuthal apertures of the crystals must vary with position along the detector

\footnotetext{
ae-mail: e.casarejos@uvigo.es
} 

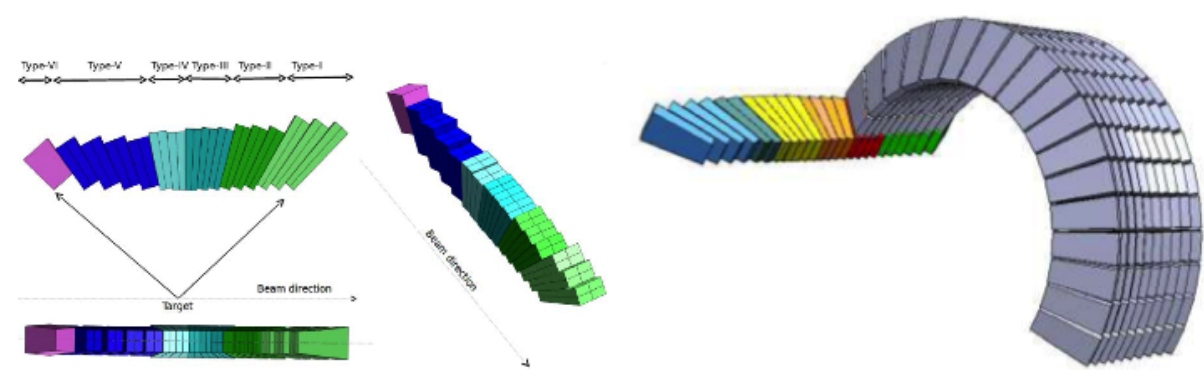

Figure 1. Left panel: three views of a row of the CALIFA BARREL segmentation. The segmentation in the polar angle determines the geometrical limit of resolution for the doppler shift correction. Right panel: the filling of the azimuthal angle is done with equal pieces per ring, covering the maximum volume to skip void spaces.

to adapt to the geometry, as well as the radial length of the crystals, selected to fit with the range of the most energetic particles. The optimum cost-effective solution for the BARREL is based on almost $2000 \mathrm{CsI}(\mathrm{Tl})$ crystals covering an angular range from 43 to 140 degrees in a compact geometry, with an internal radius of $300 \mathrm{~mm}$ that maximizes the calorimetric qualities.

The BARREL is split into two symmetric halves, which can operate either separately, or closed together, or even unpaired (shifted) in the beam direction. The functional detector consist in the active core of the $\mathrm{CsI}(\mathrm{Tl})$ crystals and the mechanical structure to hold and move the active core, based in a carbon-fibre alveolar structure, a barrel-like cover, and an external moving structure. All the necessary front-end electronics, cabling, gas piping, temperature and slow control, etc. will be included.

The Technical Design Report (TDR) of the BARREL [4] was approved by the external experts committee of FAIR by the end of 2012. The construction of CALIFA will start in 2013 with the so called DEMONSTRATOR [5]. This is a structure based in CALIFA mechanical solutions, covering about $20 \%$ of the detector active elements, and available for physics experiments in 2014 at GSI. CALIFA is expected to be ready for commissioning in 2016.

\section{CF-composite structure for the core}

The overall constrains for the active core made of almost $2000 \mathrm{CsI}(\mathrm{Tl})$ crystals include: i/ a robust and safe structure ii/ a minimum of structural material, and iii/ a tight definition of the static positioning and orientation of the crystals. The design solution is an alveolar structure made of carbon fibre composites (CF) to support the crystals. Extensive studies based in GEANT4 simulations and mechanical calculations with finite elements analysis in ANSYS [6] were performed, and guided the engineering design to reach an optimum version of the CF-structure. The design optimization was focused on the one hand, on the optimal segmentation to reduce the Doppler broadening without damaging the calorimetric properties. On the other hand, the R\&D worked with the structural materials with the motto 'the less, the better' to minimize the energy losses in the passive material, which is critic for the energy resolution of the protons.

In the figure 1 we show the segmentation of the CF-structure in both polar and azimuthal angles. The CF-structure has 16 rings, each made of 32 pieces that minimize the empty spaces. The segmentation was optimized according to a balance between a limited influence in the geometric component of the resolution [7], and a limited number of elements. The self-supported honeycomb structure is mechanically optimized, with a wall thickness below $0.3 \mathrm{~mm}$. The ratio of the mass of the CF-structure 

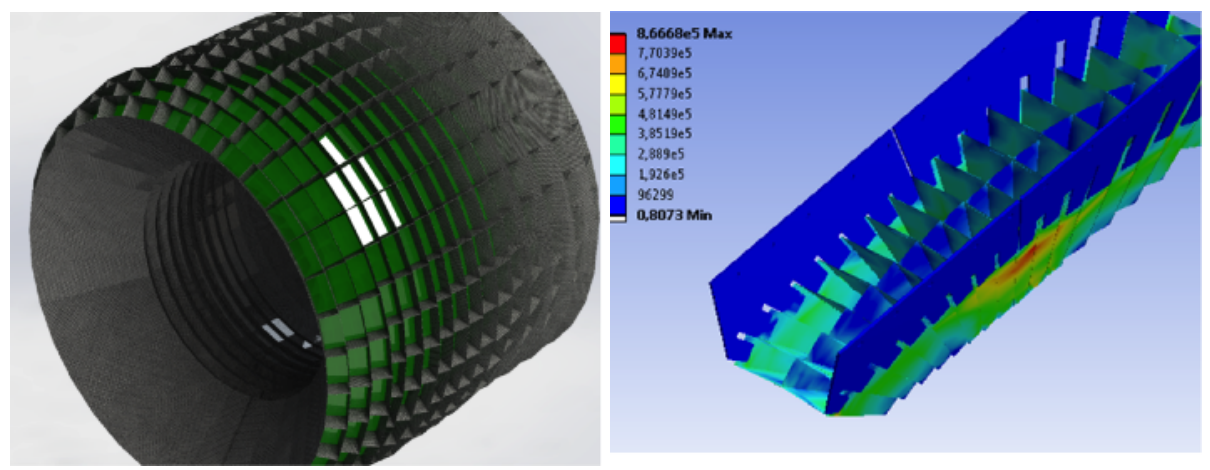

Figure 2. Left panel: view of the whole set of CF whose pieces form a self-supported honeycomb structure. The wall thickness of the pieces is below $0.3 \mathrm{~mm}$. Right panel: the assembly of the CF structure and the pieces with flaps that hold it at the upper part of the honeycomb walls, required detailed mechanical calculations. The figure show the results of one of those evaluations using a finite elements model.

and the mass of the active crystals (about $1300 \mathrm{Kg}$ ) is below $0.7 \%$. In the figure 2 (left panel) we show a view of the whole CF-stucture. The structure is calculated and designed to be hold by the upper part of the honeycomb walls. A dedicated piece with flaps holds the structure and fit it to the next structure, being this assembly a key of the whole structure. In the figure 2 (right panel) we show the result of one of the structural calculations of a segment of the CF-structure.

\section{Cover structure}

A modular cover structure holds the CF-structure, and connects with the external structure. In the figure 3 (left panel) we show a drawing of the COVER structure, built up with similar modules.

This structure closes the active core to make a gas- and light-tight volume. That is mandatory due to the sensitivity and hygroscopicity of $\mathrm{CsI}(\mathrm{Tl})$ crystals. Moreover, the response of the crystal and photosensor is temperature dependent. To cope with the requirements the active volume will be filled with nitrogen renewed in a closed loop at controlled temperature. In the outer surface, the COVER holds the pre-amplifier units, and distributes the external and internal refrigeration gas piping. In the figure 3 (right panel) we show the assembly of the CF-structure, the COVER and the elements on its surface.

\section{External structure}

The external structure must support the active core, allowing for the partition of the system in two autonomous and symmetric halves. The two blocks are closed in the nominal configuration of CALIFA. Other configurations are possible: a single half alone, and also both halves set unpaired with a longitudinal shift between them to allow for a clearance of the forward angles of one side.

The EXTERNAL structure is gantry-like, with arms that clip the COVER structure, and with additional platforms and rails. The EXTERNAL structure allows for movements in X-Y-Z quasi independently. The opening of the halves is achieved by sliding the base platforms over rails, set at about 15 degrees, also moving away the structure from the big dipole magnet placed next to it in the future installation. The same platforms slide in the longitudinal direction, with an independent system, 

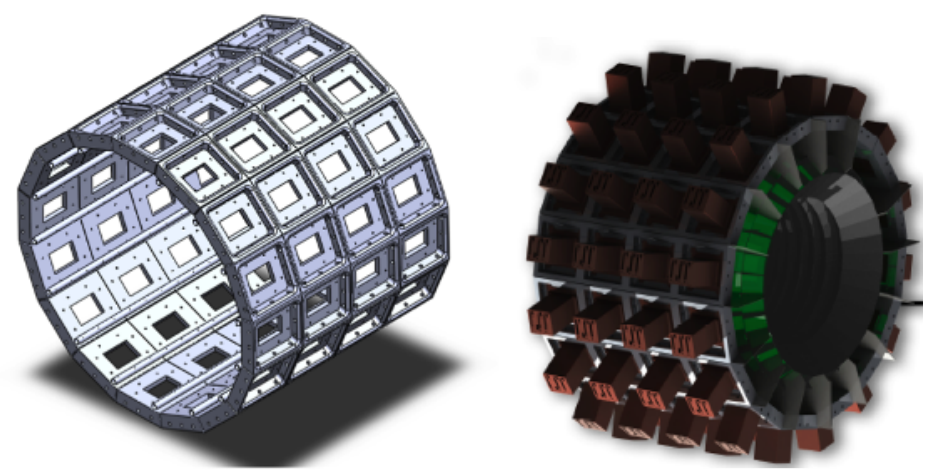

Figure 3. Left panel: the COVER structure, made of similar modules, makes a rigid and robust structure that supports the active core inside. Right panel: the cover makes a closed volume for the active core, and supports on its surface the units of the pre-amplification stage and temperature control piping.
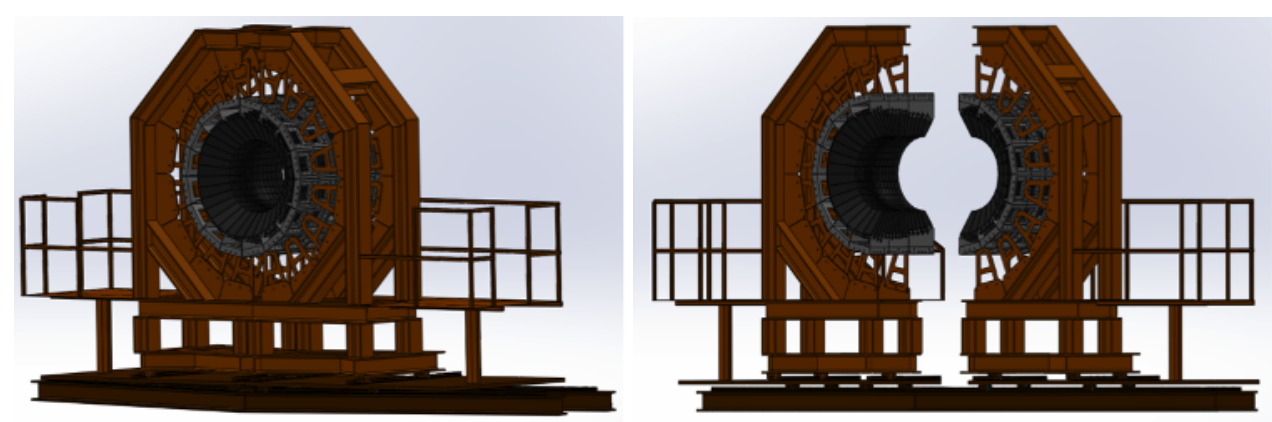

Figure 4. The EXTERNAL structure supports the CF-structure with arms that join the COVER and the gantry. Moving platforms and rails allow for opening the two halves of CALIFA, as well as other movements, allowing for the fine fitting of the core, a clearance of the forward angles, as well as helping in the setup of the future Forward EndCap.

to allow the relative shifting between the halves. These movements also help for the fine fitting of the core, a clearance of the forward angles, as well as helping in the setup of the future Forward EndCap. In the figure 4 we show two views of the whole assembly in its nominal position (left), and with the two blocks opened (right).

\section{References}

[1] R3B, http://www.gsi.de/r3b

[2] FAIR, An International Facility for Antiproton and Ion Research, http://www.fair-center.eu

[3] Helmholtzzentrum für Schwerionenforschung GmbH, Germany.

[4] http://www.fair-center.eu/for-users/experiments/nustar/nustar-documents/technical-design-reports.html

[5] E. Casarejos et al., Design and construction of the structure of the DEMONSTRATOR..., in this proceedings.

[6] ANSYS is a trademark for ANSYS software, http://www.ansys.com

[7] H. Alvarez-Pol et al., Nucl. Inst. and Meth. B 266 4616-4620 (2008). 